\title{
On the 1/f noise in the UV solar spectral irradiance
}

\author{
Costas A. Varotsos - Irina Melnikova • Maria N. Efstathiou • \\ Chris Tzanis
}

Received: 6 October 2012 / Accepted: 28 January 2013 /Published online: 13 February 2013

(C) Springer-Verlag Wien 2013

A major shortcoming of many climate studies on long-range dependence is the inference of long-term memory from climate data without considering that the power-law scaling needs to be established and a simple exponential decay of the autocorrelation function has to be rejected (Efstathiou and Varotsos 2010; Maraun et al. 2004; Varotsos 2005a; Varotsos and Kirk-Davidoff 2006; Varotsos et al. 2003a, b, 2005, 2006, 2007, 2008, 2009, 2013).

Very recently, Varotsos et al. (2013), studying the highresolution observations of the spectral solar incident flux (SIF) reaching the ground and the top of the atmosphere, concluded that SIF versus ultraviolet wavelengths (WL) exhibit $1 /$ f-type power-law correlations. This result was based on the slope (i.e., $1.02 \pm 0.02$ ) of the $\log -\log$ plot of the root mean square fluctuation function $F_{d}(\tau)$ of SIF versus the WL segment size $\tau$, after the application of the detrended fluctuation analysis (DFA).

However, the reader of the paper by Varotsos et al. (2013) may argue that the afore-mentioned power-law scaling should be established by employing the two criteria suggested by Maraun et al. (2004), notably the rejection of the exponential decay of the autocorrelation function and the constancy of "local slopes" in a certain range towards the low frequencies.

In this respect, we herewith plot the profile of the power spectral density (i.e., the distribution of the variance over

C. A. Varotsos $(\bowtie) \cdot$ M. N. Efstathiou $\cdot$ C. Tzanis

Climate Research Group, Division of Environmental Physics and Meteorology, Faculty of Physics, University of Athens, University Campus Bldg. Phys. V,

Athens 15784, Greece

e-mail: covar@phys.uoa.gr

I. Melnikova

Laboratory for Atmospheric Physics, Research Center for Interdisciplinary Environmental Cooperation (INENCO RAS), Russian Academy of Sciences, St. Petersburg, Russia frequency) versus $\log \tau$ for the detrended SIF-WL data set (depicted in Fig. 1a). This plot shows that in a WL scale range from $\log \tau \approx 0.3$ (given that the measurement step is 0.05 , the value of $\left.\tau \approx 10^{0.3} \times 0.05 \approx 0.1 \mathrm{~nm}\right)$ to maximally $\log \tau \approx 1.46\left(\tau \approx 10^{1.46} \times 0.05 \approx 1.4 \mathrm{~nm}\right)$, the power spectral density is better fitted exponentially, while for larger scales, it turns into an algebraically (power-law) fit (i.e., satisfying thus the first criterion of Maraun et al. (2004) for the rejection of the exponential fit). On the other hand, we apply the method of local slopes, suggested by Maraun et al. (2004), in order to examine the establishment of the $1 / \mathrm{f}$ noise in the UV solar spectral irradiance. Since the single straight line of the DFA plot for the detrended SIF-WL data set that established in the whole range of scales may be biased, we evaluate the local slopes of $\log F_{d}(\tau)$ versus $\log \tau$ detecting for constancy in a sufficient range. Along these lines, we first fit a straight line to $\log F_{d}(\tau)$ versus $\log \tau$ within a specific window of WL scale range. This window is then shifted successively over all calculated scales $\tau$. At this point, it should be noted that Maraun et al. (2004) state verbatim: "Choosing the optimal window size, one has to trade bias for variance: For small windows, the bias is small, but the variability renders the interpretation difficult, whereas for large windows, the variance is reduced at the cost of a biased estimate of $a$." Figure 1b illustrates the local slope $a$ versus $\log \tau$ for two different window sizes. In addition, we compute the standard deviations, $s_{\mathrm{a}}$, of each estimated local slope over all the scales, along with the error bounds of each local slope $a$, defined as $a \pm 1.96 \cdot s_{\mathrm{a}}$. Inspection of Fig. $1 \mathrm{~b}$ shows that for small scales, the variance of the local slopes is low, while for large scales, the variance is increased with $a$ values reaching to a constant threshold $(a \approx 0.92)$ at a range that coincides with that in Fig. 1a, where the power-law behavior prevails (establishing thus the long-range dependence). Similar results were obtained by considering other windows. 
Fig. 1 a Power spectral density of the detrended SIF-WL (in milliwatt per square meter per nanometer) versus $\log \tau$, with the power-law (black line) and the exponential (gray line) fit $\left(y=40524482 x^{5.65}\right.$ with $R^{2}=$ 0.93 and $y=28924 \mathrm{e}^{6.475 x}$ with $R^{2}=0.82$, respectively). b Local slopes of the $\log F_{d}(\tau)$ versus $\log \tau$ calculated within a window of 18 points (dashed gray line) and 20 points (solid black line) for the detrended SIF-WL data set. The error bars indicate the corresponding $1.96 \cdot s_{\mathrm{a}}$-intervals of the slopes over all the considered scales a

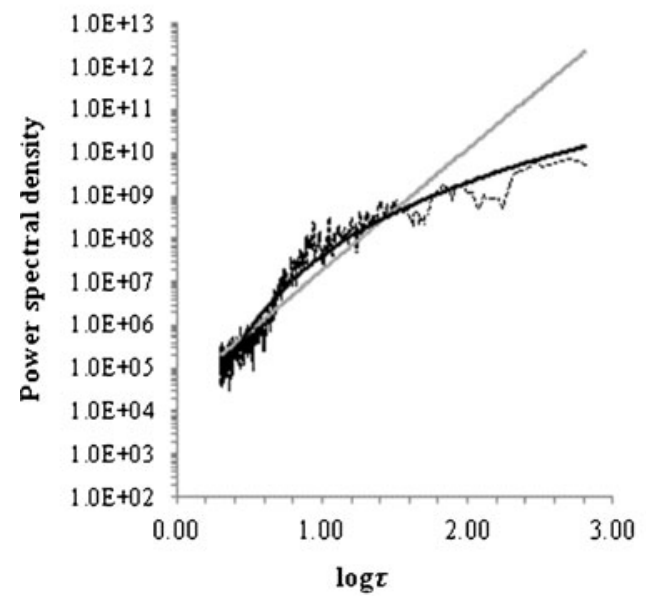

b

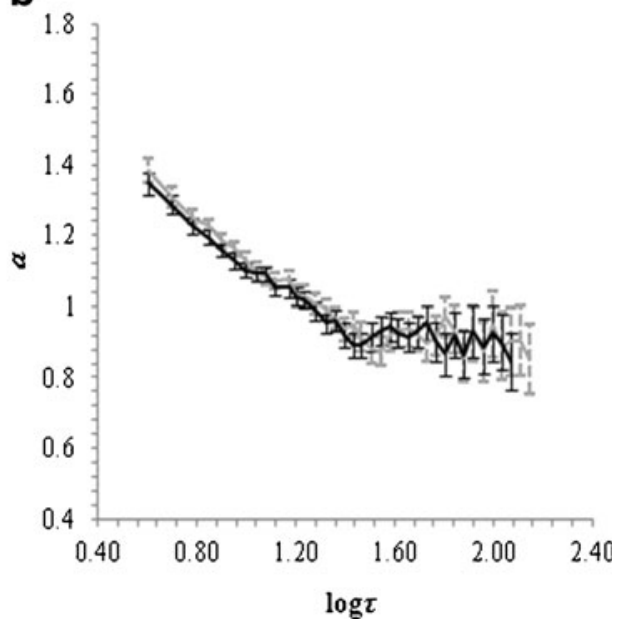

The fulfillment of the two criteria of Maraum et al. (2004) shown above ensures the 1/f-type power-law correlations in SIF versus WL suggested by Varotsos et al. (2013). Interestingly, the WL range found in Fig. $1 \mathrm{~b}$ is of the same order of magnitude (i.e., WL lags between about 0.2 and $30 \mathrm{~nm}$ ) with that suggested by Varotsos et al. (2012), fitting better to the WL separation of the major Fraunhofer lines in the UV $(299.4,302.1,336.1,358.1,382.0,393.4,396.8$, and $410.2 \mathrm{~nm}$ ). These separations range from 2.7 to $34.0 \mathrm{~nm}$ and are of the same order of the wavelength interval of the $1 / \mathrm{f}$ behavior mentioned above. The latter is of crucial importance for the UV impacts to the atmosphere and biosphere (Alexandris et al. 1999; Efstathiou et al. 1998; Kondratyev et al. 1995; Kondratyev and Varotsos 1995, 1996; Feretis et al. 2002; Varotsos et al. 1998; Varotsos 2005b; Cracknell and Varotsos 2007; Tzanis et al. 2008; Ziemke et al. 2000).

\section{References}

Alexandris D, Varotsos C, Kondratyev KY, Chronopoulos G (1999) On the altitude dependence of solar effective UV. Phys Chem Earth PT C 24:515-517

Cracknell AP, Varotsos CA (2007) Fifty years after the first artificial satellite: from Sputnik 1 to ENVISAT. Int J Remote Sens 28:2071-2072

Efstathiou MN, Varotsos C (2010) On the altitude dependence of the temperature scaling behavior at the global troposphere. Int $\mathrm{J}$ Remote Sens 31:343-349

Efstathiou M, Varotsos C, Kondratyev KY (1998) An estimation of the surface solar ultraviolet irradiance during an extreme total ozone minimum. Meteorol Atmos Phys 68:171-176

Feretis E, Theodorakopoulos P, Varotsos C, Efstathiou M, Tzanis C, Xirou T, Alexandridou N, Aggelou M (2002) On the plausible association between environmental conditions and human eye damage. Environ Sci Pollut R 9:163-165
Kondratyev KY, Varotsos CA (1995) Atmospheric ozone variability in the context of global change. Int $\mathrm{J}$ Remote Sens 16:1851-1881

Kondratyev KY, Varotsos CA (1996) Global total ozone dynamicsimpact on surface solar ultraviolet radiation variability and ecosystems. Environ Sci Pollut R 3:205-209

Kondratyev KY, Pokrovsky OM, Varotsos CA (1995) Atmospheric ozone trends and other factors of surface ultraviolet radiation variability. Environ Conserv 22:259-261

Maraun D, Rust HW, Timmer J (2004) Tempting long-memory-on the interpretation of DFA results. Nonlin Process Geophys 11:495-503

Tzanis C, Varotsos C, Viras L (2008) Impacts of the solar eclipse of 29 March 2006 on the surface ozone concentration, the solar ultraviolet radiation and the meteorological parameters at Athens, Greece. Atmos Chem Phys 8:425-430

Varotsos C (2005a) Power-law correlations in column ozone over Antarctica. Int J Remote Sens 26:3333-3342

Varotsos C (2005b) Airborne measurements of aerosol, ozone, and solar ultraviolet irradiance in the troposphere. J Geophys Res 110:10. doi:10.1029/2004JD005397

Varotsos C, Kirk-Davidoff D (2006) Long-memory processes in ozone and temperature variations at the region 60 degrees S-60 degrees N. Atmos Chem Phys 6:4093-4100

Varotsos CA, Chronopoulos GJ, Cracknell AP, Johnson BE, Katsambas A, Philippou A (1998) Total ozone and solar ultraviolet radiation, as derived from satellite and groundbased instrumentation at Dundee, Scotland. Int J Remote Sens 19:3301-3305

Varotsos PA, Sarlis NV, Skordas ES (2003a) Long-range correlations in the electric signals that precede rupture: further investigations. Phys Rev E. doi:10.1103/PhysRevE.67.021109

Varotsos PA, Sarlis NV, Skordas ES (2003b) Attempt to distinguish electric signals of a dichotomous nature. Phys Rev E. doi:10.1103/PhysRevE.68.031106

Varotsos C, Ondov J, Efstathiou M (2005) Scaling properties of air pollution in Athens, Greece and Baltimore, Maryland. Atmos Environ 39:4041-4047

Varotsos CA, Ondov JM, Cracknell AP, Efstathiou MN, Assimakopoulos MN (2006) Long-range persistence in global aerosol index dynamics. Int J Remote Sens 27:3593-3603 
Varotsos C, Assimakopoulos MN, Efstathiou M (2007) Technical note: long-term memory effect in the atmospheric $\mathrm{CO}_{2}$ concentration at Mauna Loa. Atmos Chem Phys 7:629-634

Varotsos CA, Milinevsky G, Grytsai A, Efstathiou M, Tzanis C (2008) Scaling effect in planetary waves over Antarctica. Int J Remote Sens 29:2697-2704

Varotsos C, Efstathiou M, Tzanis C (2009) Scaling behaviour of the global tropopause. Atmos Chem Phys 9:677-683
Varotsos CA, Melnikova I, Efstathiou MN, Tzanis C (2013) $1 / \mathrm{f}$ noise in the UV solar spectral irradiance. Theor Appl Climatol 111(3-4):641-648. doi:10.1007/s00704012-0697-8

Ziemke JR, Chandra S, Herman J, Varotsos C (2000) Erythemally weighted UV trends over northern latitudes derived from Nimbus 7 TOMS measurements. J Geophys Res D6:73737382 